\title{
Medievalista
}

Online

$25 \mid 2019$

Número 25

\section{Roland Scriptoris ou de Lisbonne: un médecin, un professeur et un clerc portugais au $\mathrm{XV}^{\mathrm{e}}$ siècle}

Rolando Scriptoris or of Lisbon: a Portuguese Physician, Professor and Clergyman of the Fifteenth Century

\section{Mário Farelo}

\section{OpenEdition}

\section{Journals}

Édition électronique

URL : http://journals.openedition.org/medievalista/1750

DOI : 10.4000/medievalista. 1750

ISSN : 1646-740X

\section{Éditeur}

Instituto de Estudos Medievais - FCSH-UNL

\section{Référence électronique}

Mário Farelo, «Roland Scriptoris ou de Lisbonne: un médecin, un professeur et un clerc portugais au XVe siècle », Medievalista [En ligne], 25 | 2019, mis en ligne le 17 mars 2019, consulté le 01 mai 2019. URL : http://journals.openedition.org/medievalista/1750; DOI : 10.4000/medievalista.1750

Ce document a été généré automatiquement le 1 mai 2019.

(c) IEM 


\title{
Roland Scriptoris ou de Lisbonne: un médecin, un professeur et un clerc portugais au $\mathrm{XV}^{\mathrm{e}}$ siècle
}

\author{
Rolando Scriptoris or of Lisbon: a Portuguese Physician, Professor and \\ Clergyman of the Fifteenth Century
}

Mário Farelo

\section{NOTE DE L'ÉDITEUR}

Data recepção do artigo / Received for publication: 02-11-2018

Data aceitação do artigo / Accepted in revised form: 07-12-2018

1 La Bibliothèque d'Ajuda à Lisbonne conserve, sous la cote 52-XII-18, un traité de physiognomie du XV siècle depuis longtemps connu de l'historiographie portugaise ${ }^{1}$. Malheureusement, le parcours de son auteur a demeuré assez longtemps ignoré de l'historiographie portugaise, alors que les grandes lignes de son trajet personnel, académique et professionnel ont été dégagées depuis des décennies par les travaux d'Ernest Wickersheimer ${ }^{2}$, Lynn Thorndike ${ }^{3}$, John Bartier ${ }^{4}$, Thérèse Charmasson ${ }^{5}$, Danielle Jacquart $^{6}$, Raphael de Keyser ${ }^{7}$, Jan Veenstra ${ }^{8}$, Jacques Paviot ${ }^{9}$ ou Malte Prietzel ${ }^{10}$. En effet, ce n'est que très récemment que ces contributions ont été mises à profit par l'historiographie portugaise ${ }^{11}$, sans toutefois en epuiser la richesse informative desdites synthèses ou des travaux spécialisés sur sa production scientifique ${ }^{12}$.

2 Le présent travail prétend ainsi revisiter les diverses facette de la vie de ce lusitanien, notamment ses origines, son action comme suppôt universitaire et officier ducale, ainsi que son parcours bénéficial ${ }^{13}$. 


\section{Ses origines}

3 Comme pour bien d'autres hommes médiévaux, aucun document connu à ce jour fourni la date exacte de la naissance de Roland Scriptoris. Toutefois, à la lumière des indications avancées par son parcours et par la date de son décès, quelque part dans la seconde moitié de la décennie $1470^{14}$, il faut envisager une naissance vers la fin du XIV e siècle ou le début de la centurie suivante ${ }^{15}$.

4 Par ailleurs, il se déclare "phisicus Ulixbonensis" dans la dédicace de deux de ces ouvrages, l'une de physiognomie (Reductorium phisionomie, vulgo Physiognomie) et l'autre d'arithmétique (Aggregatorium sive compedium artis arismetrice). Pour les historiens portugais, cette mention est la preuve de sa naturalité lisbonnaise ${ }^{16}$. Cependant, celle-ci ne fait pas unanimité, puisque longtemps a été suivie la version d'Ernest Wickersheimer, selon laquelle cette expression signifie seulement que Roland Scriptoris fut un français qui exerça une activité médicale à Lisbonne ${ }^{17}$. Cette thèse fut plus tard remise en question par Thérèse Charmasson en citant une lettre de légitimation émise par le duc de Bourgogne où le nom de sa mère évoque une origine portugaise (Beatriz Gonçalves) ${ }^{18}$. La réponse de l'énigme se trouve dans deux suppliques qu'il dirigea au Saint Père où il se déclare "clerc du diocèse de Lisbonne" ${ }^{19}$. Le fait que ce type de source indique normalement l'origine géographique du suppliant, par le biais de la mention du diocèse dans lequel ce dernier est né, permet de dissiper en définitif tout doute sur la naturalité portugaise de Roland Scriptoris ${ }^{20}$.

5 En ce qui concerne ses origines familiales, la lettre de légitimation révèle qu'il était fils "naturel, bâtard et illégitime" des alors décédés Jacques Lescrivain et de Beatrix Ton Salve, célibataire $^{21}$. Nous ignorons tout sur la vie de son père. Probablement il s'agit d'un personnage lié à la cour de Bourgogne $e^{22}$ qui a peut-être rencontré la portugaise Beatriz Gonçalves dans la région lisbonnaise après un voyage à but commercial ou autre ${ }^{23}$. Il est vraisemblable qu'elle eut suivit Jacques et s'ait fixé à Bruges, où il existait une importante communauté portugaise ${ }^{24}$. C'est d'ailleurs dans cette ville que nous retrouvons pour la première fois Roland, lorsqu'il est désigné, le 12 octobre 1418, dans la qualité de citoyen de la ville en compagnie de son frère Olivier ${ }^{25}$. Il faut ajouter que cette appartenance à Bruges se poursuivra au long de toute sa vie, soit dans le service communal de la ville ${ }^{26}$, soit dans l'occupation de bénéfices ecclésiastiques y établis ${ }^{27}$ ou soit encore dans l'aide à sa famille, notamment à son frère Jean, à sa sœur et à son beau-frère qui vivaient, vers 1440 dans une maison appartenant au décanat qu'il détenait à saint-Donatien de Bruges ${ }^{28}$.

6 Malgré son insertion familiale brugeoise, une bonne partie de sa vie a été passée à Paris, plus précisément à l'Université, à une époque où le recrutement de ses membres issus des pays urbanistes avait diminué, mais sans jamais s'arrêter pour complet ${ }^{29}$.

\section{Le parcours universitaire de Roland Scriptoris}

\subsection{Les années de formation}

7 Attestée sa fréquence à l'Université de Paris, comme nous le verrons, il nous semble peu défendable la thèse selon laquelle il aurait suivi des cours de Médecine à l'Université de Montpellier. Une idée mise de l'avant par Augusto da Silva Carvalho pour expliquer le trajet de l'auteur de la Physiognomia conservée à la Bibliothèque d'Ajuda, celle-ci entre en 
collision avec le manque de sources et le peu de cas attestés d'une double insertion dans ces deux facultés de Médecine ${ }^{30}$. Ainsi, si l'on excepte une mention qui ne fait pas l'unanimité pour l'année 1406-1407 - faisant de lui le recteur du studium generale ${ }^{31}$-, la première mention sûre à son trajet universitaire date de 1419-1420, lorsqu'il paya seize sous au doyen de la Faculté de Médecine pour faire sceller trois cédules ${ }^{32}$. Il est alors désigné de maître, ce qui laisse croire qu'il aurait alors terminé ces études dans la faculté «préparatoire» des Arts avec l'acquisition d'une maîtrise ${ }^{33}$, comme c'était l'usage à l'époque, à partir de l'observation des statuts facultaires ${ }^{34}$.

8 Roland prétendait ainsi attester sa participation aux actes scolaires, le premier pas vers la requête future du grade de bachelier, qu'il acquière pendant le premier semestre de 1422 35 .

9 Les deux ans suivants furent remplis à satisfaire les leçons et les disputationes exigées par les statuts $^{36}$. Après cette période, il s'est préparé pour accéder à la licence, laquelle, heureusement pour lui, tomba dans ce même année jubilaire de 1423-1424 $4^{37}$. Dans la réunion facultaire du 4 mars 1424, Roland a présenté une somme de cinquante-sept mois sans inclure les mois de l'année universitaire en cours ${ }^{38}$. En obéissant aux statuts, il a pu alors obtenir le tant désiré grade académique, non pas sans débourser le montant de trois francs et huit deniers pro bursis sue licentie et dix-huit deniers pour sa première leçon en tant que régent de la faculté (inceptione magisterii in medicina) ${ }^{39}$. Nous ne savons pas la date d'obtention du doctorat dans cette discipline, une donnée confirmée d'ailleurs uniquement par des sources non-facultaires ${ }^{40}$.

\subsection{La régence d'une chaire à la Faculté de Médecine de Paris}

10 Par le biais des actes des réunions de la Faculté de Médecine consignées par le doyen ou son substitut, il est sûr que Roland aurait enseigné comme actu regens pendant un total de dix-sept ans ${ }^{41}$. À la lumière de ces informations, il est clair que sa carrière de professeur doit être mise en relation avec la présence anglo-bourguignonne à Paris entre 1418 et $1436^{42}$. En effet, très souvent les allusions aux obligations de Roland Scriptoris en tant que régent révèlent les liens qu'il maintient avec son protecteur, le duc de Bedford. En guise d'exemple, rappelons le parrainage de Jean Kline, un protégé dudit duc, qui le choisit comme domino commiso le $1^{\mathrm{er}}$ mars $1432^{43}$. De surcroît, c'est sans doute dans la double qualité d'universitaire et de périt en médecine, au service du duc de Bedford, qu'il assista en compagnie des maîtres Guillaume de Gardinis, Egidius Canivet et Guillaume de Camera à la sixième session du procès de Jeanne d'Arc, entre le 9 janvier et le 30 mai $1431^{44}$.

11 À notre connaissance, rien n'est arrivé à nos jours de son enseignement, à l'instar de nombreux médecins universitaires parisiens qui voyaient dans le fait de ne pas écrire la protection de leur savoir et, par ce fait même, des secrets de leur facultét5. Il était néanmoins très intéressé par les sciences, non seulement celles du corps, mais aussi celles en relation avec les arts divinatoires et dont il ne manquerait pas d'inclure dans son activité médicale. Trois indices permettent cette idée. D'une part, les trois ouvrages qui sont arrivés jusqu'à nous: un traité déjà mentionné de physiognomie intitulé Reductorium phisionomie, vulgo Physiognomie, un autre de geomancie (Compilatorium sive aggregatorium totius artis geomancie ad astronomiam redacte et un troisième d'arithmétique (Aggregatorium sive compedium artis arismetrice $)^{46}$. Il n'est pas ici la place pour discuter de la valeur scientifique de ces ouvrages. Cependant, ces thèmes demeurent en marge des curricula en vigueur à la faculté, même si les auctoritates mentionnées dans ses travaux soient celles 
couramment employées dans le milieu universitaire parisien, en faisant foi aux spécialités de ces questions ${ }^{47}$. En second lieu, un autre argument demeure les controverses qu'il eût à ce sujet, dont celle survenue en 1437 entre Roland et Laurent Musche, maître-ès-Arts et bachelier en Théologie sur la rédaction de l'almanach de cette année-là pour les jours favorables aux saignées et à l'application de laxatifs ${ }^{48}$. En dernier lieu, cet engouement pour les sciences se manifeste également dans emprunts de livres d'astrologie, de physique et de médicine en mettant à profit la richesse bibliographique des grandes bibliothèques parisiennes, comme en témoigne l'inventaire de ces prêts consignés dans le tableau I ${ }^{49}$.

Tableau I - Les prêts bibliothécaires en faveur de Roland Scriptoris

\begin{tabular}{|c|c|c|}
\hline Date & Cuvre & Bibliothèque \\
\hline $\begin{array}{ll}5 & \text { août } \\
1430 & \end{array}$ & $\begin{array}{l}\text { Trois premières décades des Histoire de Tite- } \\
\text { Live }\end{array}$ & Chapitre de Notre-Dame de Paris ${ }^{50}$ \\
\hline 1432 & $\begin{array}{l}\text { Commenta Alberti super diversos libros physice } \\
\text { d'Albert le Grand }\end{array}$ & $\begin{array}{l}\text { Nation allemande de l'Université } \\
\text { de Paris }{ }^{51}\end{array}$ \\
\hline $\begin{array}{l}1^{\text {er }} \text { avril } \\
1435\end{array}$ & De judiciis astronomie & Collège de Sorbonne ${ }^{52}$ \\
\hline $\begin{array}{l}15 \text { avril } \\
1435\end{array}$ & l'Antidotarium de Jean de saint-Amand & Collège de Sorbonne $\mathrm{e}^{53}$ \\
\hline $\begin{array}{l}15 \text { avril } \\
1435\end{array}$ & Secretum de Oculis de Pierre d'Espagne & Collège de Sorbonne ${ }^{54}$. \\
\hline
\end{tabular}

Outre ses intérêts scientifiques, découlant de son activité enseignante et courtisane, Roland était appelé à accumuler sa fonction de régent avec une participation active au sein de l'Université et, tout particulièrement, au sein de la Faculté que l'a reçu comme régent.

\subsection{Le fonctionnaire universitaire}

Il n'est pas impossible que Roland Scriptoris ait bénéficié des charges dans structure organisationnelle de l'alma mater parisienne avant son passage à la faculté de Médicine, en jugeant par les références qui suggèrent son rôle en tant que procureur de la nation française ${ }^{55}$ et recteur de l'Université ${ }^{56}$. Nonobstant, c'est à la faculté de Médicine que son activité bureaucratique se cristallisera, au long de deux périodes apparemment assez distinctes. La première s'étale dès 1424 , date à laquelle il acquiert sa licence en Médecine, jusqu'à la fin de la décennie suivante. Pendant ce temps, Roland joint à ses responsabilités de maitre-régent une activité assez soutenue dans sa faculté, soit dans la qualité de son doyen, à quatre reprises ${ }^{57}$, soit comme son représentant en divers procès au Parlement de Paris ${ }^{58}$. Quoi qu'il en soit, Roland ne tarda pas à s'affirmer dans ce milieu. Comme preuve, son élection au décanat annuel tout de suite pendant l'année 1424-142559, justifiant alors que certaines des réunions facultaires soient alors effectuées chez lui, à la porte saint- 
Severin, près de la taverne appelée L'écu de France ${ }^{60}$. Il sera plus tard le doyen de la faculté entre $1427-1428$ et $1429-1430^{61}$.

Après cette période et jusqu'à sa sortie du milieu universitaire parisien, la documentation universitaire disponible sur lui concerne, presque en exclusif, ses attributions de maîtrerégent. Cette chute potentielle dans son engagement dans sa faculté pourrait se relier avec l'évolution elle-même de la vie universitaire: après les premières années d'affirmation au sein de la corporation, les années suivantes sont celles de reconnaissance.

Roland y sera en place jusqu'en $1442^{62}$. Sa sortie de la corporation n'est pas sans relation avec les changements soufferts à partir de ce moment par l'Université: des grèves, de la peste et l'absentéisme qui conduisit à son effondrement et à l'intervention finalement du cardinal d'Estouteville en $1452^{63}$. Désormais, il sera tout acquis au service des Grands ${ }^{64}$.

\section{Le service du duc de Bedford}

a pratique de la médecine théorique constituait l'une des options professionnelles qui s'offraient aux médecins universitaires dans les cours royales, ducales et comtales. Ces spécialistes réunissaient très souvent dans l'art de fixer des diagnostiques par la récitation des auctoritates, de même que la maîtrise de certains connaissances astrologiques pouvaient fournir une aide précieuse dans la prise de décisions par lerus patrons. Le médecin universitaire était, bien des fois, un astrologue, un fait qui lui valait un rôle politique dans la Cour où il séjournait. Nous pensons que Roland s'inscrit dans cette fonction courtisane que l'on vient de décrire grossièrement.

Les premières indications dont nous disposons sur son appartenance au corps de physiciens du duc de Bedford datent le 1423-1425, période qui constitue d'ailleurs la fourchette chronologique admise pour la rédaction de son Aggregatorium sive compedium artis arismetrice, œuvre dédiée elle-même à Bedford en guise de rétribution pour l'intercession ducale dans l'obtention d'un canonicat et d'une prébende à la SainteChapelle de Paris ${ }^{65}$. Il subsiste néanmoins des doutes sur les raisons qui ont justifié son engagement par le duc. Sans doute, plus que le lien de cousinage entre celui-ci et l'infanta Isabelle, future duchesse de Bourgogne, il faut y voir l'intérêt pour le duc des compétences scientifiques de Roland. Or, ses attributions de médecin ducal nous échappent, à cause de l'absence de données provenant des documents de la pratique ou des chroniques. Toutefois, il est sûr que Roland s'est engagé à satisfaire l'intérêt bibliophiles du Duc, puisqu'il composa, à sa demande, la Physiognomia et son traité d'arithmétique ${ }^{66}$.

18 La maitrise de ces sujets fit de lui un naturel reviseur des traductions scientifiques élaborées à la demande de Bedford, comme en témoigne le cas de Jean Tourtier. Ce chirurgien, dûment autorisé à pratiquer après avoir suivi des cours à la Faculté de Médicine de Paris, mais où ne semble pas avoir acquis aucun grade, copia à Rouen, en 1429-1430, les Aphorismes d'Hippocrate avec le commentaire de Galien de Martin de SaintGilles par l'ordre du Duc. Or, dans son explicit, il énumère les trois maîtres qui pourraient revoir son travail. Un de ceux-là est précisément Roland l'Escripvain qu'il appelle "mom maistre maistre"67.

Guidés par ces maigres faits, nous sommes tentés de penser que la pratique des techniques divinatoires fut l'action principale accomplie par Roland auprès du duc de 
Bedford. En effet, nous n'ignorons pas qu'il se vivait alors une période d'augmentation en importance de l'astrologie judiciaire, où des savants capables de projeter des événements et des conjonctures futures, ainsi que de présenter des options lors de la prise de décisions, amenaient une valeur ajoutée aux gouvernants. A cet effet, le manuscrit latin 7443, conservé à la Bibliothèque Nationale de Paris, contient un curieux dossier astrologique daté de 1426-1427 et destiné au duc de Bedford. Bien qu'il ne se recèle pas aisement le nom de son auteur, l'analyse interne de son contenu fait prévoir une intervention important de notre Roland Scriptoris au niveau de sa conception. Ce même manuscrit contient par ailleurs des nouvelles sur ses capacités astrologiques. Cette fois, qui l'affirme c'est nul autre que Simon Boesmare, l'un des juges du différend que Roland eût sur l'établissement de l'almanach en 1437 et qui prononça une sentence qui lui fut défavorable à propos de l'horoscope du couronnement de Charles VII ${ }^{68}$. Sans que l'on sache la portée de cette décision, il semble que sa crédibilité n'ait pas été grandement affectée, puisqu'il est engagé par le duc de Bourgogne, après la mort du duc de Bedford en septembre 1435.

\section{Le service de la maison de Bourgogne}

$\mathrm{Au}$ contraire du cas précédant, les sources disponibles prouvent, hormis tout doute, les liens de service que Roland maintiendra avec la maison de Bourgogne, notamment avec Philippe, le Bon et son fils, Charles, le Téméraire. Il a aussi exercé son activité de médecin de façon plus ponctuelle auprès de sa compatriote, la duchesse Isabelle ${ }^{69}$.

21 Séjournant le plus souvent à Bruges, qui était alors dans la mouvance bourguignonne, maître-régent de la faculté de Médicine, son cas rassemblait bien ceux d'autres médecins ducaux comme Jacques Sacquespée, Jacques Despars, Eustache Cailleu et Jean Cotterau, avec lesquels il partageait une insertion universitaire à Paris. Son passage de la maison de Bedford à celle de Bourgogne semble avoir été direct, d'autant plus que Nicolas de la Horbe, médecin et astrologue de Jean Sans Peur et de Philipe le Bon, avait décédé vers cette même époque ${ }^{70}$.

Or, cette hypothèse s'avère très vraisemblable dans la mesure où les très riches sources financières bourguignonnes registent son nom dans la liste des gages payés aux officiers de la maison ducale, dès le mois de juin $1437^{71}$. Ces documents mentionneront désormais très régulièrement le nom de Roland jusqu'en 1469 - avec des différents périodes d'absence qui peuvent varier de quelques jours à quelques mois - comme il possible de voir, à partir de la liste de 541 entrées établié à partir des données mises en ligne dans la base de données Prosopographica burgundica ${ }^{72}$. Par ailleurs, cette chronologie est passable de confirmation à partir de deux documents émanant de la Cour des Comptes de Lille qui résument les sommes versées à Roland par la maison bourguignonne. Le premier indique une pension annuelle de cent francs qui lui fut octroyée par lettre patente du duc, du 13 octobre 1438, avec des effets rétroactifs dès le 9 avril 1437 et qui lui fut versée jusqu'en décembre $1461^{73}$. La seconde, du 14 mai de cette dernière année, consacre l'obligation de lui remettre au quotidien la somme de dix-huit soldes, ce qui semble avoir été remplié jusqu'en $1467^{74}$. Cette dernière date doit correspondre au passage au service de Charles, le nouveau duc ${ }^{75}$.

Nous recensons très peu de informations sur les services médicaux fournis par Roland à la maison de Bourgogne. Sans doute son rôle allait au-delà de la surveillance des plats ingérés et des symptômes de maladies, de l'aide dispensée à l'habillement du Prince ${ }^{76}$ et 
de la vente de produits pour l'amélioration de la santé du Duc ${ }^{77}$. Étant donné sa capacité astrologique, il constituait, à l'instar de ses collègues, un important conseiller politique qui obtint des bénéfices et dont la considération à la Cour qui n'échappa pas à l'observation contemporaine du chroniqueur bourguignon Olivier de la Marche:

Le Duc a six docteurs médecins, et servent iceulx à visiter la personne et l'état de la santé du Prince. Et quand le Duc est à la table, iceulx médecins sont derrière le bancq et voient de quoy et de quelz metz et viandes l'on sert le Prince et luy conseillent à leur adviz lesquelles viandes luy sont prouffitables; ils peuvent à toutes les heures entrer en la chambre du Prince et sont gens si notable, si bons si grans clercs qu'ilz peuvent être à beaucoup de conseilz huchiez et appelez; ils ont plat à court comme le premier sommelier, mais ilz n'ont point de chambre ordinaire $^{78}$.

\section{Sa carrière bénéficiale}

Le parcours bénéficiale de Roland Scriptoris a été sans doute marqué par la nécessité de pallier son défaut d'illégitimé et de franchir les divers rangs dans les ordres ecclésiastiques. Le premier a été nécessaire pour accéder et pour maintenir les bénéfices qu'il avait reçus par collation de l'ordinaire comme il se voit par l'analyse de notre tableau II.

Tableau II - Les bénéfices ecclésiastiques de Roland Scriptoris

\begin{tabular}{|c|c|c|}
\hline Bénéfices & $\begin{array}{l}\text { Première } \\
\text { référence }\end{array}$ & $\begin{array}{l}\text { Dernière } \\
\text { référence }\end{array}$ \\
\hline Canonicat et prébende du chapitre de saint-Donatien de Bruges & 1422 & $\begin{array}{l}1 \text { novembre } \\
1439^{79}\end{array}$ \\
\hline Canonicat et prébende de la sainte-Chapelle de Paris & $\begin{array}{l}\text { Vers } \\
1423-1425\end{array}$ & $1435^{80}$ \\
\hline $\begin{array}{l}\text { Dispense du défaut d'illégitimité pour pouvoir recevoir deux } \\
\text { canonicats avec la réserves des respectives deux prébendes }\end{array}$ & \multicolumn{2}{|l|}{25 avril $1424^{81}$} \\
\hline $\begin{array}{l}\text { Dispense du défaut d'illégitimité pour maintenir la pluralité de } \\
\text { bénéfices: canonicat et prébende de saint-Sauveur d'Harlebeek et } \\
\text { l'église paroissiale de Sesme }\end{array}$ & \multicolumn{2}{|l|}{25 août $1427^{82}$} \\
\hline Dispense du défaut d'illégitimité & \multicolumn{2}{|c|}{25 octobre $1427^{83}$} \\
\hline $\begin{array}{l}\text { Ne réussit par l'élection dans un bénéfice à la collation de } \\
\text { l'Université de Paris }\end{array}$ & \multicolumn{2}{|c|}{24 janvier $1428^{84}$} \\
\hline Église paroissiale d'Eden & $\begin{array}{ll}\text { Avant mars } \\
1428\end{array}$ & $\operatorname{mars} 1428^{85}$ \\
\hline Chapellenie dans l'hôpital de Notre-Dame de Nazereth à Bruges & \multicolumn{2}{|l|}{ mars $1428^{86}$} \\
\hline Supplique de deux canonicats et respectives deux prébendes & \multicolumn{2}{|l|}{11 avril $1428^{87}$} \\
\hline
\end{tabular}




\begin{tabular}{|l|l|l|}
\hline $\begin{array}{l}\text { Décanat et prébende annexe du chapitre de saint-Donatien de } \\
\text { Bruges }\end{array}$ & $\begin{array}{l}1 \text { novembre } \\
1439\end{array}$ & 7 avril $1476^{88}$ \\
\hline Canonicat, prébende et archidiaconat du chapitre d'Arras & $\begin{array}{l}23 \text { septembre } \\
1439\end{array}$ & $1475^{89}$ \\
\hline
\end{tabular}

Nous voyons que la question de l'illégimité ne fut résolue qu'en 1427 par la concession de la nécessaire dispense par Martin $\mathrm{V}$ pour accéder aux ordres mineurs et à un bénéfice ecclésiastique sans cure (alors que la legitimation de la part du Duc n'a lieu que beaucoup plus tard, en 1460). Fort de ce privilège, il peut alors espérer gravir les échelons du sousdiaconat (concédé en 1431 par l'évêque de Paris), le diaconat en 1440 et l'ordination presbytérale, octroyée en 1442 par l'évêque de Troyes $^{90}$.

L'analyse de ce parcours permet de voir qu'il acquière le gros de ces bénéfices pendant la décennie 1420, lorsqu'il accède aux grades en médicine et qu'il débute sa carrière de maître-régent à Paris. Curieusement, il jouit seulement d'un bénéfice issu de cette région, puisque tous les autres remettent pour une insertion bourguignonne vraisemblement proche, notamment à Bruges et dans le diocèse de Tournai ${ }^{91}$. Ce n'est que plus tard, après avoir entré au service des Ducs de Bourgogne, qu'il arrive à obtenir des bénéfices plus payants (la doyenneté de saint-Donatien de Bruges et l'archidiaconat d'Arras), sans doute avec l'aide de ces nouveaux protecteurs ${ }^{92}$.

Ainsi, son parcours bénéficial illustre particulièrement bien un des traits de la politique ecclésiastique poursuivit par les ducs de Bourgogne, en termes du contrôle des provisions à des personnes de leur famille et de leurs clientèles des bénéfices des évêchés établies dans les territoires sous sa domination ou même sous domination de ses voisins ${ }^{93}$. Mais, il illustra aussi comment cette accumulation bénéficiale peut-être néfaste. En réalité, la jouissance de ces bénéfices de fut pas ne tout repos, dans la mesure où son manque de assiduité préjudicait la satisfaction de ses obligations liturgiques et juridictionnelles dans les chapitres d'Arras ${ }^{94}$ et de saint-Donatien de Bruges ${ }^{95}$.

Roland put profiter d'un ensemble important de rendements ecclésiastiques qui, ajoutés aux gages reçus dans la qualité de médecin ducale, fournissaient vraisemblablement ses plus importantes sources de financement. Cependant, il pouvait à l'occasion lancer main d'autres revenus moins réguliers par la mise à disposition de ses connaissances médicales à ceux qui en seraient en mesure de se les payer, comme des villes ou des institutions privées ${ }^{96}$. Somme toute, lorsque la mort de Roland le surprend, quelque part vers le début de la décennie 1470, il est sans doute un homme vieux ${ }^{97}$.

Il avait fait du chemin: né à Lisbonne, il passa une bonne partie en France et dans les États bourguignons. Il a sans doute eu du mal à cacher son tempérament latin, révélé parfois de manière sui generis, comme la journée où il frappa le recteur de l'Université en pleine réunion de la nation allemande ${ }^{98}$. Probablement sa punition n'était pas si grand, car il était un éminent personnage universitaire et de sa faculté. Il l'avait servie étant que son doyen, il l'a représentée au Parlement ou même dans le procès de Jeanne d'Arc. Lorsqu'il passa en Bourgogne, il laissa derrière une Université qui ne fonctionnait plus, accablée qu'elle était par les grèves et qui déconsidérait de plus en plus la figure du régent en médicine. Mais aussi, peut-être, il laissa derrière lui une institution qui ne voulait peutêtre plus de lui. Il trouvera refuge parmi ceux qui avaient besoin de lui. Du coup, il ne manqua pas de renouer avec ces origines lusitaniennes. 
La présence universitaire que nous venons d'évoquer fit de lui un expert, comme en témoignent les ouvrages qu'il écrivit. Cette autorité, mêlée avec une certaine sagesse, fut sans doute appréciée par ceux qui pouvaient employer un maitre de la médicine et un expert dans arts divinatoires. La récompense de ces services se déclina dans les privilèges, les bénéfices et les gages qu'il a reçus. De l'homme qui a vécu pendant la plus grande partie du XV siècle, nous ne connaitrons jamais les angoisses, les succès, les envies, les déboires. Ce qui reste, c'est donc sa figure publique. Le Roland que nous avons la chance de connaître, ici et là, n'est nul autre que le fonctionnaire, le courtisan, le clerc et, surtout, le scientifique.

\section{BIBLIOGRAPHIE}

Fontes impressas

CATALOGUE générale des manuscrits des bibliothèques publiques des départements. Vol. III. Manuscrits de la bibliothèque Saint-Omer. Paris: Imprimerie Nationale, 1861.

COMMENTAIRES de la Faculté de Médecine de l'Université de Paris (1395-1516). Ed. Ernest Wickersheimer. Paris: Imprimeries Nationales, 1915.

COSTA, António Domingues de Sousa - Monumenta Portugaliae Vaticana. Vol. IV. Braga-Porto: Editorial Franciscana, 1970.

DENIFLE, Henrich; CHATELAIN, Emile - Chartularium Universitatis Parisiensis. Vol. IV. Parisiis: ex typis fratrum Delalain, 1897.

DENIFLE, Henrich et al. - Auctarium Chartularii Universitatis Parisiensis: Liber procuratorum nationis Anglicanae (Alemanniae). Paris: Apud H. Didier, 1937.

Der Briefwechsel Karls des Kuhnen (1433-1477). Ed. Werner Paravicini et al. Frankfurt: Lang, 1995. 2 vols.

DU BOULAY, César Égasse - Remarques sur les bedeux de l'Université. Paris: Pierre de Bresche et Jacques Laize-de Bresche, 1670.

DU BOULAY, César Égasse - Historia Universitatis Parisiensis. Vol. V. Paris: Pierre de Bresche et Jacques Laize-de Bresche, 1670.

FOPPENS, J. P. - Compendium chronologicum episcoporum Brugensium. Brugis: Typis Jacobi Beernaerts in via Breydelia, 1731.

GAILLIARD, Edward ; GILLODTS-VAN SEVEREN, Louis - Inventaire des archives de la ville de Bruges. Bruges: E. Gailliard, 1876

GREVE, Anke et al. - Comptes de l'argentier de Charles le Téméraire. Vol. I. Paris: De Boccard, 2001.

LE GLAY, A. et al. - Inventaire sommaire des archives départementales antérieures à 1790, Nord: Archives civiles, Série B: Chambre des Comptes de Lille. Lille: Imprimerie de L. Danel, 1899-1906. 
MARCHE, Olivier de la - Mémoires d'Olivier de la Marche, maître-d'hotel et capitaine des gardes de Charles de Téméraire. Ed. Henri Beaune et Jules D'Arbaumont. Vol. IV. Paris: Librairie Renouard, 1888.

MARICHAL, Robert - Le Livre des prieurs de Sorbonne (1431-1485). Paris: Aux amateurs des Livres, 1987.

PARMENTIER, R. A. - Indices op de Brugsche poorterboeken. Deel. I. Brugge: Desclée de Brouwer, 1938.

PROCÈS de condamnation de Jeanne d'Arc. Texte, traduction et notes de Pierre Champion. Vol. I. Paris: Honoré Champion, 1920-1921. Vols. I et II.

PROCÈS de condamnation de Jeanne d'Arc. Ed. Pierre Tisset et Yvonne Lanhers. Paris: C. Klincksieck, 1960. Vols. I et II.

SANDERUS, Antonius - Flandria illustrata, sive provinciae ac comitatus hujus description. Vol. II. Bruxellis: Apud Carolum de Vos et Joannem-Baptistam de Vos, Fratres, 1735.

SOMMÉ, Monique - La correspondance d'Isabelle de Portugal, duchesse de Bourgogne (1430-1471). Ostfildern: Jan Thorbecke Verlag, 2009.

VIEILLARD, Jeanne; JULLIEN DE POMMEROL, Marie-Henriette - Le registre de prêt de la bibliothèque du Collège de Sorbonne de la Sorbonne (1402-1536). Paris: CNRS-Editions, 2000.

Estudos

AVELAR, Helena - Vir sapiens dominabitur astris. Astrological knowledge and Practices in the Portuguese Medieval Court (King João I to king Afonso V). Lisbonne: Faculté de Sciences Sociales et Humaines de l'Université Nouvelle de Lisbonne, 2011. Mémoire de Maîtrise.

AVELAR, Helena; RIBEIRO, Luís Campos - "Fisionomia e astrologia: o manuscrito de Rolando de Lisboa". in BARREIRA, Catarina Fernandes (Ed.) - Luz, cor e ouro. Estudos sobre manuscritos iluminados. Lisbonne: Biblioteca Nacional de Portugal - Instituto de Estudos Medievais, 2016, pp. 235-248.

BARTIER, John - Légistes et gens de finances au XV'esiècle. Les Conseillers des ducs de Bourgogne Philippe le Bon et Charles le Téméraire. Bruxelles: J. Duculot, 1955.

BAVEYE KOUIDRAT, Laurie - Exercer la médecine en milieu princier au XVème siècle. L'exemple de la Cour de Bourgogne (1363-1482). Lille: Université Lille 3 - Charles de Gaulle, 2015. Thèse de Doctorat.

BEAUJOUAN, Guy - "The Place of Nicolas Chuquet in a Typology of Fifteenth-Century Arithmetics". in HAY, Cynthia (Ed.) - Mathematics from Manuscripts to print 1300-1600. Oxford: Clarendon Press, 1988, pp. 73-88.

BERLIÈRE, Ursmer - Monasticon Belge. Vol. III. Bruges: Centre Nationale de Recherches d'histoire religieuse, 1978.

BOUDET, Jean-Philippe - Lire dans le Ciel. La bibliothèque de Simon de Phares, astrologue du XVe siècle. Bruxelles: Centre d'Études des Manuscrits, 1994.

BOUDET, Jean-Philippe; CHARMASSON, Thérèse - "Une consultation astrologique princière en 1427". in Comprendre et maîtriser la nature au Moyen age. Mélanges d'histoire et des sciences offerts à Guy Beaujouan. Genève: Librairie Droz, 1994, pp. 255-278.

CARLIER, Myriam - "La politique des autorités envers les bâtards dans les Pays-Bas bourguignons". in PREVENIER, Walter et BOONE, Marc (Dirs.) - Finances publiques et finances privées au bas moyen âge. Public and Private Finances in the Late Middle Ages. Louvain e Apeldoorn: Garant, 1996, pp. 203-218. 
CARVALHO, Augusto da Silva - História da Medicina portugueza. Lisbonne: Imprensa Nacional, 1929.

CARVALHO, Augusto da Silva - "Notice sur deux manuscrits de medicine". in VI Congrès international d'histoire de la Médecine. Leyde-Amsterdam, 18-23 juillet 1927. Anvers: Imprimerie de Vlijt, 1929, p. 152-155.

CHARMASSON, Thérèse - "Sciences et techniques divinatoires au XV siècle: Roland l'Ecrivain, médecin, astrologue et géomancien". in Positions des Thèses. Paris: École Nationale des Chartes, 1973, pp. 27-31.

CHARMASSON, Thérèse - “L'établissement d'un almanach médical pour l'année 1437". in Comptes-rendus du $99^{e}$ Congrès national des sociétés savantes. Besançon, 1974. Section des sciences. Fascicule V. Paris: Bibliothèque Nationale, 1976, pp. 217-234.

CHARMASSON, Thérèse - "Roland l'Écrivain, médecin des ducs de Bourgogne". in Comptes-rendus du $101^{e}$ Congrès national des sociétés savantes. Lille, 1976. Section des sciences. Fascicule III. Paris: Bibliothèque Nationale, 1976, pp. 21-32.

CHARMASSON, Thérèse - "L'Arithmétique de Roland l'Éscrivain et le Quadripartitum numerorum de Jean de Murs". Revue d'Histoire des Sciences 31/2 (1978), pp. 171-176.

CHARMASSON, Thérèse - Recherches sur une technique divinatoire: la géomancie dans l'Occident médiévale. Paris-Genève: Librairie H. Champion-Librairie Droz, 1980.

CHATELAIN, Émile - "Notes sur quelques tavernes fréquentées par l'Université de Paris aux XIV et $\mathrm{XV}^{\mathrm{e}}$ siècles”. Bulletin de la Société de l'histoire de Paris et de l'île-de-France 25 (1898), pp. 87-109.

COSTA, António Domingues de Sousa - "Mestre Afonso Dinis, médico e secretário de D. Afonso IV, Professor na Universidade de Paris”. Itinerarium 3/16-17 (1957), pp. 370-417, 510-606.

COURTENAY, William J. - "The Registers of the University of Paris and the Statutes against the Scientia Occamica". Vivarium 29/1 (1991), pp. 13-49.

DE LA FONS-MELICOCQ, Alexandre - "Les calligraphies et les manuscrits des cathédrales d'Arras, de Tournai, et de l'abbaye de Saint-Bertin". Revue du Nord de la France 1 (1854), pp. 21-24.

DELISLE, Léopold - Le cabinet des manuscrits de la Bibliothèque Nationale. Vol. I. Paris: Imprimerie Nationale, 1881 Vols I-III.

DOORSLAER, G. Van - “Traitement de la lithiase rénale en 1490”. Yperman: Bulletin de la Société belge de l'histoire de la médecine. Bulletin der belgische vereeniging voor geschiedenis der geneeskunde 1 (1924), pp. 122-127.

DROLET, Sébastien - La nation anglo-allemande de l'Université de Paris pendant la domination anglobourguignonne (1418-1438). Montréal: Faculté des Arts et des Sciences de l'Université de Montréal, 2007. Mêmoire de Maîtrise.

DUBRULLE, Henry - "Les bénéficiaires des diocèses d'Arras, Cambrai, Thérouanne, Tournai sous le pontificat d'Eugène IV d'après les documents conservés aux Archives d'État, à Rome (suite)". Analectes pour servir à l'histoire ecclésiastique de la Belgique, troisième série, t. 3, 33 (1907), pp. 57-72, 313-328, 433-448; 34 (1908), pp. 233-296.

FARELO, Mário - La peregrinatio academica portugaise vers l'Alma mater parisienne, XII ${ }^{e}-X V^{e}$ siècles. Montréal: Faculté Des Arts et des Sciences de l'Université de Montréal, 1999. Mémoire de Maîtrise.

FAYEN, A. - "Note sur un registre des Annates de la province de Reims sur Eugène IV (années 1431 à 1441)". Analectes pour servir à l'histoire ecclésiastique de la Belgique 35 (1909), pp. 261-284. 
FRANKLIN, Alfred - Les anciennes bibliothèques de Paris. Vol. I. Amsterdam: G. Th. Van Heusden, 1868.

FREIRE, Anselmo Braamcamp - "Maria Brandoa, a do Crisfal. (Cap. II - A Feitoria de Flandres)". Archivo Historico Portuguez 6 (1908), pp. 322-442.

GARRIGUE, Laurent - "Les professions médicales à la fin du Moyen Âge. L'exemple des praticiens parisiens devant le Parlement de Paris au XV siècle”. Bibliothèque de l'École des chartes 156/2 (1999), pp. 317-367.

GUESNON. A. - "Le Cartulaire de l'évêché d'Arras, manuscrit du XIII siècle avec addition jusqu'au milieu du $\mathrm{XVI}^{\mathrm{e}}$, analysé chronologiquement". Mémoires de l'Académie des Sciences, Lettres et Arts d'Arras. $2^{\mathrm{e}}$ série, 33 (1902).

JACQUART, Danielle - Le milieu médical en France du XII ${ }^{e}$ au XV $\mathrm{XV}^{e}$ siècle. Deuxième supplément au Dictionnaire d'Ernest Wickersheimer en annexe. Genève-Paris: Droz-Champion, 1981.

JACQUART, Danielle - "Hippocrates en français, le Livre des Amphorismes de Martim de SaintGille (1262-1363)”. in JACQUART, Danielle - Les voies de la science grecque. Genève: Librairie Droz, 1997, pp. 241-329

JACQUART, Danielle - La Médecine médiévale dans le cadre parisien, XIVe-XVe siècle. Paris: Fayard, 1998.

KEYSER, Raphael de - "Individuel en collectief boekenbezit bij de kanunniken van het SintDonaaskapittel te Brugge tijdens de late meddeleeuwen". Archives et Bibliothèques de Belgique 42/3-4 (1971), pp. 347-378; 43/1-2 (1972), pp. 141-175; 43/3-4 (1972), pp. 493-516.

KEYSER, Raphael de - Het Sint-Donaaskapittel te Brugge (1350-1450): Bijdrage tot de studie van hogere geestelijkheed tijdens de late middeleeuwen. Louvain: Université de Louvain, 1972. 3 vols. Thèse de Doctorat.

KEYSER, Raphael de - "Het boekenbezit en het boekengebruik in de seculiere kapittels van de zuidelijke nederlanden tijdens de middleleeuwen". Archives et bibliothèques de Belgique 11 (1974), pp. 9-68.

KEYSER, Raphael de - “Scrivers (De Scrivere, Lescrivain, Lescripvant, L’Escriveur, Scriptoris, Escritor), Roland, kanunnik, raadsheer en geneesher". in DUVERGER, Jozef (Ed.) - Nationaal Biografisch Woordenboek. Vol. VI. Bruxelles: Paleis der Academiën, 1974, cols. 854-857.

KRUSE, Holger - Hof, Amt und Gage. Die täglichen Gagenlisten des burgundischen hofes (1430-1467) und fer erste Hofstaat Karls des Kühnen (1456). Bonn: Bouvier Verlag, 1996.

LABORDE, Le Comte de - Les ducs de Bourgogne. Études sur les lettres, les arts et l'industrie pendant le $X V^{e}$ siècle. Vol. I. Paris: Plon Frères, éditeurs, 1852.

LEITÃO, Henrique - “O primeiro matemático português? Rolando de Lisboa (fl. 1424)". Jornal de Matemática Elementar 292 (2011), pp. 1-5.

LEME, Margarida Ortigão Ramos Paes. "Os Leme - um percurso familair de Bruges a Malaca”. Sapiens: História, Património e Arqueologia 0 (2008), pp. 51-83.

MEIRINHOS, José Francisco - "A atribuição a Petrus Yspanus das Sententia super libro de physionomia de Guillalmus Hispanus, no manuscrito Vaticano, Urb. Lat. 1392". Mediaevalia. Textos e Estudos 7-8 (1995), pp. 329-359.

MIRANDA, Flávio - "Commerce, conflits et justice: les marchands portugais en Flandres à la fin du Moyen Âge”. Annales de Bretagne et des Pays de l'Ouest 117/1 (2010), pp. 193-208. 
MOLLAT, Michel - "Quatre missives d'Isabelle de Portugal, duchesse de Bourgogne". Revista Portuguesa da História 11-12 (1964), pp. 1-9.

MOREAU, Édouard de - "Les familiers des ducs de Bourgogne dans les canonicats dans les anciens Pays-Bas". in Mélanges d'histoire offerts à León Van der Essen (Miscellanea historica in honorem Leonis Van der Essen. Universitatis Catholicae in oppido Lovaniensi iam Annos XXV professoris. Bruxelles: Éditions universitaires, 1947, pp. 429-437.

MOYSE, Gérard - “Les suppliques médiévales: documents lacunaires, documents répétitifs?”. in FOSSIER, Lucie; VAUCHEZ, André; VIOLANTE, Cinzio (Dirs.) - Informatique et histoire médiévale. Communications et débats de la Table Ronde CNRS, organisée par l'École Française de Rome et l'Institut d'Histoire Médiévale de Pise (Rome, 20-22 mai 1975). Rome: École Française de Rome, 1977, pp. 55-72.

NASCIMENTO, Aires A., et al. (Ed.) - A imagem do tempo: Livros manuscritos ocidentais. Museu Calouste Gulbenkian: Galeria de exposições temporárias, de 31 Março a 2 Julho 2000. Lisbonne: Fundação Calouste Gulbenkian, 2000.

LOUIS, Nicolas - L'exemplus en pratiques. Production, diffusion et usages des recueils d'exempla latins aux $13^{e}-15^{e}$ siècles. Namur: Université de Namur, 2013. Thèse de Doctorat.

PAQUET, Jacques - "L'immatriculation des étudiants dans les universités médiévales". in Pascua Mediaevalia. Studies voor Prof. Dr. J.M. de Smet. Louvain: Universitaire pers Leuven, 1983, pp. 159-171.

PAVIOT, Jacques - Portugal et Bourgogne au $\mathrm{XV}^{e}$ siècle: recueil de documents extraits des archives bourguignonnes. Lisbonne-Paris: Commission Nationale pour les Commémorations des Découvertes Portugaises - Centre Culturel Calouste Gulbenkian, 1995.

PAVIOT, Jacques - "Les Portugais à Bruges au XV ${ }^{e}$ siècle”. Arquivos do Centro Cultural Calouste Gulbenkian 38 (1999), pp. 1-122.

PEARSON, Andrea - Gender in Burgundian Devotional Art, 1350-1530. Experience, Authority, Resistance. Aldershot: Ashgate, 2005.

POULLE, Emmanuel - "Horoscopes princiers des XIV et XVe siècles". Bulletin de la société des antiquaires de France (1969), pp. 63-69.

PRIETZEL, Malte - Guillaume Fillastre der jüngere (1400/07-1473). Stuttgart: Jan Thorbecke, 2001.

RACICOT, Annie - L'Université de Paris et le pouvoir royal sous le règne de Louis XI (1461-1483). Montréal: Faculté des Arts et des Sciences de l'Université de Montréal, 2000. Mémoire de Maîtrise.

"ROLANDO de Lisboa". in CEPEDA, Isabel Vilares; FERREIRA, Teresa A. S. Duarte (Dir.) - Inventário dos códices iluminados até 1500. Vol. I. Distrito de Lisboa. Lisbonne: Secretaria de Estado da CulturaIBNL, 1994.

ROUSE, Richard H. et ROUSE, Mary A. - Illiterari et uxorati. Manuscripts and Their Makers: commercial Book Producers in Medieval Paris, 1200-1500. Vol. 2. Turnhout: Brepols, 2000.

SCHREVEL, A. C. de - Histoire du séminaire de Bruges. Vol. I. Bruges: Imprimerie A. de Zuttere-van Kersschaver, 1895.

SOMMÉ, Monique - "Isabelle de Portugal et Bruges: des relations privilégiées". Handelingen Genootschap "Société d'Emulation" Brugge, 132 (1995), pp. 261-279.

SOMMÉ, Monique - "Les Portugais dans l'entourage de la duchesse de Bourgogne Isabelle de Portugal (1430-1471)”. Revue du Nord 77/310 (1995), pp. 321-343. 
SERRÃO, Joaquim Veríssimo - Les Portugais à l'Université de Montpellier, XII ${ }^{e}$-XVII ${ }^{e}$ siècles. Paris: Centre culturel Portugais-Fondation Calouste Gulkenkian, 1971.

STRATFORD, Jenny - "The Manuscripts of John, Duke of Bedford: Library and Chapel”. in WILLIAM, Daniel (Ed.) - England in the Fifteenth Century. Wooldbridge: Boydell Press, 1987, pp. 329-350.

SZPIRGLAS, Jacques - Prosopographie des musiciens des Saintes-Chapelles de Paris (1248-ca1640) et de Bourges (1405-ca1640). Tours: Université François-Rabelais, 2015. Thèse de Doctorat.

THORNDIKE, Lynn - A History of Magic and Experimental Science during the First Thirteen Centuries of our Era. Vol. IV. New York: Columbia University Press, 1934.

TROALEN, Philippe - Charles le Téméraire, ses maladies et ses médecins. Paris: Université Paris Valde-Marne, 1981. Thèse de Doctorat.

VEENSTRA, Jan R. - Magic and Divination at the Courts of Burgundy and France. Text and Context of Laurens Pignon's Contre les divinateurs. Leiden-New York-Köln: Brill, 1998.

VERGER, Jacques - "The University of Paris at the End of the Hundred Years' War". in BALDWIN, John W.; GOLDTHWAITE, Richard A. (Eds.) - Universities and Politics. Case Studies from the Late Middle Ages and Early Modern Period. Boltimore-London: The John Hopkins Press, 1972, pp. 47-78.

VERGER, Jacques - "Que peut-on attendre d'un traitement automatique des suppliques?". in FOSSIER, Lucie; VAUCHEZ, André; VIOLANTE, Cinzio (Dirs.) - Informatique et histoire médiévale. Communications et débats de la Table Ronde CNRS, organisée par l'École Française de Rome et l'Institut d'Histoire Médiévale de Pise (Rome, 20-22 mai 1975). Rome: École Française de Rome, 1977, pp. 73-78.

VERGER, Jacques - "Examen privatum, examen publicum. Aux origines médiévales de la thèse". Mélanges de la Bibliothèque de la Sorbonne 12 (1993), pp. 15-43.

VULLIEZ, Charles; VERGER, Jacques - "Cartulaires universitaires français". in GUYOTJEANNIN, Olivier et al. (Eds.) - Les Cartulaires. Actes de la Table Ronde organisée par l'École Nationale des Chartes et le CNRS. Paris: École Nationale des chartes, 1993, pp. 423-449.

WATT, Donald E.R. - "University Clerks and Rolls of Petitions for Benefices”. Speculum 34 (1959), pp. 213-229.

WEIJERS, Olga - "Les règles d'examens dans les universités médiévales”. in HOENE, M.J.F.M.; SCHNEIDER, J.H.J.; WIELAND, G. (Eds.) - Philosophy and Learning. Universities in the Middle Ages. Leiden-New Yok-Köln: Brill, 1995, pp. 210-223.

WICKERSHEIMER, Ernest - Dictionnaire biographique des médecins en France au Moyen Âge. Vol. II. Paris: E. Droz, 1936, pp. 723-724.

ZIEGLER, Joseph - "Text and Context: On the Rise of Physiognomic Thought in the Later Middle Age". in HEN, Yitzhak (Ed.) - De Sion Exibit Lex et Verbum Domini de Hierusalem. Essays on Medieval Law, Liturgy and Literature in honour of Amnon Linder. Turnhout: Brepols, 2001, pp. 159-182.

ZIEGLER, Joseph - "Médecine et physiognomonie du XIV au début du XVI ${ }^{\mathrm{e}}$ siècle". Médiévales 46 (2004), pp. 89-108 (disponible sur http://medievales.revues.org/document805.html).

ZIEGLER, Joseph - "Philosophers and physicians on the scientific validity of Latin physiognomy, 1200-1500". Early Science and Medicine 12/3 (2007), pp. 285-312. 


\section{NOTES}

1. Roland s'est fait connaître au Portugal par Augusto da Silva Carvalho lorsqu'il publia l'incipit de cette œuvre, étant dorénavant répertorié dans des inventaires et des catalogues d'expositions publiés depuis. CARVALHO, Augusto da Silva - "Notice sur deux manuscrits de medicine". in VI Congrès international d'histoire de la Médecine. Leyde-Amsterdam, 18-23 juillet 1927. Anvers: Imprimerie de Vlijt, 1929, pp. 152-155; CARVALHO, Augusto da Silva - História da Medicina portugueza. Lisbonne: Imprensa Nacional, 1929; "Rolando de Lisboa". in CEPEDA, Isabel Vilares; FERREIRA, Teresa A. S. Duarte (Dirs.) -Inventário dos códices iluminados até 1500. Vol. I. Distrito de Lisboa. Lisboa: Secretaria de Estado da Cultura-IBNL, 1994, p. 98, nº. 96; NASCIMENTO, Aires A., et al. (Ed.) - A imagem do tempo: Livros manuscritos ocidentais. Museu Calouste Gulbenkian: Galeria de exposições temporárias, de 31 Março a 2 Julho 2000. Lisbonne: Fundação Calouste Gulbenkian, 2000, p. 15, 125.

2. WICKERSHEIMER, Ernest - Dictionnaire biographique des médecins en France au Moyen Âge. Vol. II. Paris: E. Droz, 1936, pp. 723-724.

3. THORNDIKE, Lynn - A History of Magic and Experimental Science during the First Thirteen Centuries of our Era. Vol. IV. New York: Columbia University Press, 1934, pp. 139-143, 584.

4. BARTIER, John - Légistes et gens de finances au XVe siècle. Les Conseillers des ducs de Bourgogne Philippe le Bon et Charles le Téméraire. Bruxelles: J. Duculot, 1955, pp. 383-384.

5. CHARMASSON, Thérèse - "Sciences et techniques divinatoires au XV siècle: Roland l'Ecrivain, médecin, astrologue et géomancien". in Positions des Thèses. Paris: École Nationale des Chartes, 1973, pp. 27-31; CHARMASSON, Thérèse - "Roland l'Écrivain, médecin des ducs de Bourgogne". in Comptes-rendus du $101^{e}$ Congrès national des sociétés savantes. Lille, 1976. Section des sciences. Fascicule III. Paris: Bibliothèque Nationale, 1976, pp. 21-32; CHARMASSON, Thérèse - "L'établissement d'un almanach médical pour l'année 1437". in Comptes-rendus $d u 9^{e}$ Congrès national des sociétés savantes. Besançon, 1974. Section des sciences. Fascicule V. Paris: Bibliothèque Nationale, 1976, pp. 217-234; CHARMASSON, Thérèse - "L'Arithmétique de Roland l'Éscrivain et le Quadripartitum numerorum de Jean de Murs". Revue d'Histoire des Sciences 31/2 (1978), pp. 171-176; CHARMASSON, Thérèse - Recherches sur une technique divinatoire: la géomancie dans l'Occident médiévale. ParisGenève: Librairie H. Champion-Librairie Droz, 1980, pp. 177-193; BOUDET, Jean-Philippe; CHARMASSON, Thérèse - "Une consultation astrologique princière en 1427". in Comprendre et maîtriser la nature au Moyen age. Mélanges d'histoire et des sciences offerts à Guy Beaujouan. Genève: Librairie Droz, 1994, pp. 255-278.

6. JACQUART, Danielle - Le milieu médical en France du XII ${ }^{e}$ au XV ${ }^{e}$ siècle. Deuxième supplément au Dictionnaire d'Ernest Wickersheimer en annexe. Genève-Paris: Droz-Champion, 1981, p. 264.

7. KEYSER, Raphael de - "Individuel en collectief boekenbezit bij de kanunniken van het SintDonaaskapittel te Brugge tijdens de late meddeleeuwen". Archives et Bibliothèques de Belgique 42/3-4 (1971), pp. 347-378; 43/1-2 (1972), pp. 141-175; 43/3-4 (1972), pp. 493-516; KEYSER, Raphael de - Het Sint-Donaaskapittel te Brugge (1350-1450): Bijdrage tot de studie van hogere geestelijkheed tijdens de late middeleeuwen. Louvain: Université de Louvain, 1972. Vol. III, pp. 21-23, 125-127. Thèse de doctorat; KEYSER, Raphael de - "Het boekenbezit en het boekengebruik in de seculiere kapittels van de zuidelijke nederlanden tijdens de middleleeuwen". Archives et bibliothèques de Belgique 11 (1974), pp. 9-68; KEYSER, Raphael de - "Scrivers (De Scrivere, Lescrivain, Lescripvant, L'Escriveur, Scriptoris, Escritor), Roland, kanunnik, raadsheer en geneesher". in DUVERGER, Jozef (Ed.) Nationaal Biografisch Woordenboek. Vol. VI. Bruxelles: Paleis der Academiën, 1974, cols. 854-857.

8. VEENSTRA, Jan R. - Magic and Divination at the Courts of Burgundy and France. Text and Context of Laurens Pignon's Contre les divinateurs. Leiden-New York-Köln: Brill, 1998, p. 130. 
9. PAVIOT, Jacques - Portugal et Bourgogne au XVe siècle: recueil de documents extraits des archives bourguignonnes. Lisbonne-Paris: Commission Nationale pour les Commémorations des Découvertes Portugaises - Centre Culturel Calouste Gulbenkian, 1995, p. 106.

10. PRIETZEL, Malte - Guillaume Fillastre der jüngere (1400/07-1473). Stuttgart: Jan Thorbecke, 2001, pp. 34, 66, 341, 455-456.

11. Augusto da Silva Carvalho demeura la référence sur le sujet pour les auteurs lusitaniens et ce n'est que plus récemment que les chercheurs portugais ont incorporé dans leurs textes les travaux des spécialistes ci-dessus mentionnés (SERRÃO, Joaquim Veríssimo - Les Portugais à l'Université de Montpellier, XII ${ }^{e}$ XVII ${ }^{e}$ siècles. Paris: Centre Culturel Portugais-Fondation Calosute Gulkenkian, 1971, p. 67; MEIRINHOS, José Francisco - "A atribuição a Petrus Yspanus das Sententia super libro de physionomia de Guillalmus Hispanus, no manuscrito Vaticano, Urb. Lat. 1392". Mediaevalia. Textos e Estudos 7-8 (1995), p. 344, note 39; FARELO, Mário - La peregrinatio academica portugaise vers l'Alma mater parisienne, $\mathrm{XII}^{e}-\mathrm{XV}^{e}$ siècles. Montréal: Faculté Des Arts et des Sciences de l'Université de Montréal; 1999, p. 226. Mémoire de Maîtrise; LEITÃO, Henrique - "O primeiro matemático português? Rolando de Lisboa (fl. 1424)”. Jornal de Matemática Elementar 292 (2011), pp. 1-5; CARDOSO, Adelino; OLIVEIRA, António Brás de; MARQUES, Manuel Silvério (Coord.) - Arte Médica e Imagem do Corpo: de Hipócrates ao final do século XVIII. Lisbonne: Biblioteca Nacional, 2010; CARVALHO, Helena Avelar de - Vir sapiens dominabitur astris. Astrological knowledge and Practices in the Portuguese Medieval Court (King João I to king Afonso V). Lisbonne: Faculté de Sciences Sociales et Humaines de l'Université Nouvelle de Lisbonne, 2011, p. 47. Mémoire de Maîtrise; CARVALHO, Helena Avelar de; RIBEIRO, Luís Campos - "Fisionomia e astrologia: o manuscrito de Rolando de Lisboa". in BARREIRA, Catarina Fernandes (Ed.) - Luz, cor e ouro. Estudos sobre manuscritos iluminados. Lisboa: Biblioteca Nacional de Portugal - Instituto de Estudos Medievais, 2016, pp. 235-248.

12. Notamment ZIEGLER, Joseph - "Text and Context: On the Rise of Physiognomic Thought in the Later Middle Age". in HEN, Yitzhak (Ed.) - De Sion Exibit Lex et Verbum Domini de Hierusalem. Essays on Medieval Law, Liturgy and Literature in honour of Amnon Linder. Turnhout: Brepols, 2001, pp. 159-182; ZIEGLER, Joseph - "Médecine et physiognomonie du XIv e au début du XVI siècle". Médiévales 46 (2004), pp. 89-108 (disponible sur http://medievales.revues.org/document805.html ); ZIEGLER, Joseph - "Philosophers and physicians on the scientific validity of Latin physiognomy, 1200-1500". Early Science and Medicine 12/3 (2007), pp. 285-312.

13. Dans le but d'alléger l'apparat critique du travail, nous limitons les références aux sources d'où nous avons puisé de l'information et à la bibliographie spécialisée, lorsque celle-ci procède à des analyses détaillées ou lorsqu'elle amène des nouveautés sur les faits étudiés.

14. Même si son nom disparaît des comptes des ducs de Bourgogne à partir de 1469, il était encore vivant le 7 avril 1476, date à laquelle il échange son décanat du chapitre collégiale de saint-Donatien de Bruges pour l'église de sainte-Marie de Slusis. Il s'est ensuivit un conflit qui se maintenait au moment de sa mort. FOPPENS, J.P. - Compendium chronologicum episcoporum Brugensium. Brugis: Typis Jacobi Beernaerts in via Breydelia, 1731, p. 82; KEYSER, Raphael de "Scrivers", col. 855. Cette chronologie correspond à l'hypothèse jadis soutenue par Wickersheimer en termes d'un décès vers 1477 (WICKERSHEIMER, Ernest - Dictionnaire biographique, p. 324).

15. Etant donné son cursus universitaire analysé infra.

16. Voir les travaux référés à la note 10.

17. WICKERSHEIMER, Ernest - Dictionnaire biographique, p. 724.

18. CHARMASSON, Thérèse - "Roland l'Écrivain", p. 22 où l'auteur publie cette lettre, datée du 18 septembre 1460.

19. Ces suppliques sont éditées dans COSTA, António Domingues de Sousa (Ed.) - Monumenta Portugaliae Vaticana. Vol. IV. Braga-Porto: Editorial Franciscana, 1970, pp. 263-264, ㄲo. 1189 (25 août 1427); pp. 294-295, nº. 1221 (11 avril 1428). 
20. Sur cette question, voir MOYSE, Gérard - "Les suppliques médiévales: documents lacunaires, documents répétitifs?" et VERGER, Jacques - "Que peut-on attendre d'un traitement automatique des suppliques?". in FOSSIER, Lucie; VAUCHEZ, André; VIOLANTE, Cinzio (Dirs.) - Informatique et histoire médiévale. Communications et débats de la Table Ronde CNRS, organisée par l'École Française de Rome et l'Institut d'Histoire Médiévale de Pise (Rome, 20-22 mai 1975). Rome: École Française de Rome, 1977, pp. 55-72 et 73-78 respectivement.

21. CHARMASSON, Thérèse - "Roland l'Écrivain", p. 22. Pour les processus de légitimation de Roland et de ses enfants, de même que sur la conjoncture économique subjacente à cette question, voir CARLIER, Myriam - "La politique des autorités envers les bâtards dans les Pays-Bas bourguignons". in PREVENIER, Walter; BOONE, Marc (Dirs.) - Finances publiques et finances privées au bas moyen âge. Public and Private Finances in the Late Middle Ages. Louvain e Apeldoorn: Garant, 1996, pp. 316-317.

22. Un certain Jean Lescripvain, chapelain de la duchesse de Bar, reçoit de celle-ci cent soldes parisis le 16 février 1363, ce qui le place dans la mouvance des ducs de Bourgogne. Il faut quandmême souligner que nous n'ayons pas de preuve sur les liens familiales entre celui-ci et le père de Roland. LE GLAY, A. et al. - Inventaire sommaire des archives départementales antérieures à 1790, Nord: Archives civiles, Série B: Chambre des Comptes de Lille. Lille: Imprimerie de L. Danel, 1899-1906, p. 164. 23. Sur la communauté bourguignonne à Lisbonne au XV e siècle, voir FREIRE, Anselmo Braamcamp -"Maria Brandoa, a do Crisfal. (Cap. II - A Feitoria de Flandres)". Archivo Historico Portuguez 6 (1908), pp. 322-442 et LEME, Margarida Ortigão Ramos Pães - "Os Leme - um percurso familair de Bruges a Malaca”. Sapiens: História, Património e Arqueologia 0 (2008), pp. 51-83.

24. SOMMÉ, Monique - "Les Portugais dans l'entourage de la duchesse de Bourgogne Isabelle de Portugal (1430-1471)”. Revue du Nord 77/310 (1995), p. 325. Nous avons cherché sans succès des références à sa mère dans ce travail. Sur la communauté portugaise à Bruges, voir FREIRE, Anselmo Braamcamp -"Maria Brandoa", pp. 322-422; SOMMÉ, Monique - "Isabelle de Portugal et Bruges: des relations privilégiées". Handelingen Genootschap "Société d'Emulation" Brugge, 132 (1995), pp. 261-279; PAVIOT, Jacques - "Les Portugais à Bruges au XV siècle". Arquivos do Centro Cultural Calouste Gulbenkian 38 (1999), pp. 1-122; MIRANDA, Flávio - "Commerce, conflits et justice: les marchands portugais en Flandres à la fin du Moyen Âge". Annales de Bretagne et des Pays de l'Ouest, 117/1 (2010), pp. 193-208.

25. PARMENTIER, R. A. - Indices op de Brugsche poorterboeken. Deel. I. Brugge: Desclée de Brouwer, 1938, p. 170; KEYSER, Raphael de - "Scrivers", col. 855.

26. Il a été élu comme consul de la ville à deux reprises en 1441 et en 1468. SANDERUS, Antonius Flandria illustrata, sive provinciae ac comitatus hujus description. Vol. II. Bruxellis: Apud Carolum de Vos et Joannem-Baptistam de Vos, Fratres, 1735, pp. 26-27.

27. Voir la section infra.

28. KEYSER, Raphael de - "Scrivers", col. 854.

29. FARELO, Mário - La peregrinatio académica, pp. 61-68, 222-226.

30. SERRÃO, Joaquim Veríssimo - Les Portugais à l'Université, p. 68. Danielle Jacquart mentionne seulement dix-huit cas entre les XIII ${ }^{\mathrm{e}}$ et $\mathrm{XV}^{\mathrm{e}}$ siècles (JACQUART, Danielle - La Médecine médiévale dans le cadre parisien, XIV ${ }^{e}-X V^{e}$ siècle. Paris: Fayard, 1998, p. 156).

31. Voir infra.

32. COMMENTAIRES de la Faculté de Médecine de l'Université de Paris (1395-1516). Ed. Ernest Wickersheimer. Paris: Imprimeries Nationales, 1915, p. 114. La cédule était un document privé que l'auteur pouvait faire authentiquer par les autorités universitaires pour attester qu'il avait assisté et avait participé aux actes prescrits par les statuts de la faculté (PAQUET, Jacques "L'immatriculation des étudiants dans les universités médiévales". in Pascua Mediaevalia. Studies voor Prof. Dr. J. M. de Smet. Louvain: Universitaire pers Leuven, 1983, p. 160).

33. Il se déclare magister in artibus dans les suppliques envoyées au pape en 1427-1428; dans l'acte de sa deuxième élection au décanat de sa faculté; dans la recette générale de 1438-1439 des ducs 
de Bourgogne ou dans l'explicit de son traité de géomancie (COSTA, António Domingues de Sousa - Monumenta, pp. 263-264; COMMENTAIRES de la Faculté de Médecine, p. 143; LABORDE, Le Comte de - Les ducs de Bourgogne. Études sur les lettres, les arts et l'industrie pendant le XVe siècle. Vol. I. Paris: Plon Frères, éditeurs, 1852, p. 355, no. 1207).

34. À l'époque où Roland entre à la Faculté de Médicine de Paris, l'obligation pour les nouveaux étudiants d'avoir été gradués au préalable avec la licence ou la maîtrise en Arts n'était pas encore en place, une fois que cela a eu lieu seulement le 1 juillet 1426. DENIFLE, Henrich; CHATELAIN, Emile - Chartularium Universitatis Parisiensis. Vol. IV. Parisiis: ex typis fratrum Delalain, 1897, p. $545, \mathrm{n}^{\circ} .2274$.

35. Le livre de registre des doyens de la faculté détaille tout le processus et les sommes déboursées par Roland (COMMENTAIRES de la Faculté de Médecine, pp. 120, 122, 123). Pour le déroulement de ces examens effectués à deux temps, voir VERGER, Jacques - "Examen privatum, examen publicum. Aux origines médiévales de la thèse". Mélanges de la Bibliothèque de la Sorbonne 12 (1993), pp. 15-43; WEIJERS, Olga - "Les règles d'examens dans les universités médiévales". in HOENE, M. J. F. M.; SCHNEIDER, J. H. J.; WIELAND, G. (Eds.) - Philosophy and Learning. Universities in the Middle Ages. Leiden-New Yok-Köln: Brill, 1995, pp. 210-223 et JACQUART, Danielle - La Médecine médiévale, pp. 130-146.

36. Pour cette question, voir JACQUART, Danielle - La Médecine médiévale, pp. 135-138.

37. En effet, ce grade n'était délivré que chaque deux ans. Le processus d'attribution de la Licence suivait les mêmes étapes du baccalauréat avec la différence que, dans ce dernier cas, les examinateurs étaient tous les maîtres régents de la Faculté de Médecine et que le temps nécessaire pour l'octroi du grade était plus grand. COSTA, António Domingues de Sousa - "Mestre Afonso Dinis, médico e secretário de D. Afonso IV, Professor na Universidade de Paris". Itinerarium 3/16-17 (1957), p. 381.

38. COMMENTAIRES de la Faculté de Médecine, p. 132.

39. COMMENTAIRES de la Faculté de Médecine, p. 132.

40. CHARMASSON, Thérèse - "Roland l'Écrivain", p. 22.

41. COMMENTAIRES de la Faculté de Médecine, pp. 134, 136, 139-140, 141, 145, 149, 150, 153, 154, 156, 158, 162, 163, 166, 167, 169-170, 171; 437, 446, 461, 468, 486, 500, 548-549, 556, 563, 574, 593, 601, $607,614,629$. Il n'existe pas de liste de régents pour les années 1428-1429 et 1441-1442. Son nom a été rayé de la liste de 1442-1443 et il ne régit plus la chair (vacat quia non continuavit regentiam) l'année suivante.

42. Pour les relations entre l'Université et cette nouvelle élite dirigeante, voir VERGER, Jacques "The University of Paris at the End of the Hundred Years' War". in BALDWIN, John W.; GOLDTHWAITE, Richard A. (Eds.) - Universities and Politics. Case Studies from the Late Middle Ages and Early Modern Period. Boltimore-London: The John Hopkins Press, 1972, p. 59; RACICOT, Annie L'Université de Paris et le pouvoir royal sous le règne de Louis XI (1461-1483). Montréal: Faculté des Arts et des Sciences de l'Université de Montréal, 2000. Mêmoire de Maîtrise; DROLET, Sébastien - La nation anglo-allemande de l'Université de Paris pendant la domination anglo-bourguignonne (1418-1438). Montréal: Faculté des Arts et des Sciences de l'Université de Montréal, 2007. Mêmoire de Maîtrise.

43. Les Commentaires énoncent d'autres activités du régent, comme la participation dans une disputatio. COMMENTAIRES de la Faculté de Médecine, pp. 151, 163.

44. PROCÈS de condamnation de Jeanne d'Arc. Texte, traduction et notes de Pierre Champion. Vol. I. Paris: Honoré Champion, 1920-1921, Vol. I, p. 69; vol. II, p. 62; PROCÈS de condamnation de Jeanne d'Arc. Ed. Pierre Tisset et Yvonne Lanhers. Paris: C. Klincksieck, 1960, Vol. I, p. 90; vol. II, pp. 408-409.

45. Pour une caractérisation des tâches des maîtres-régents à l'époque, voir VERGER, Jacques - “ Examen privatum", p. 28.

46. Voir les autres contributions pour les divers manuscrits existants sur chacune de ses œuvres. 
47. CHARMASSON, Thérèse - "Roland l'Écrivain", pp. 28-29.

48. Cette controverse est d'ailleurs paradigmatique pour justifier la pratique astrologique dans le milieu universitaire parisien dans la première moitié $\mathrm{du} \mathrm{XV}^{\mathrm{e}}$ siècle. Voir, pour tous, CHARMASSON, Thérèse - "L'établissement d'un almanach", pp. 217-234.

49. Concernant encore ce sujet, il faut ajouter la notice d'un achat à maître Jean Relier, chanoine de saint-Barthélémy de Béthune, d'un volume d'exempla, appelé Liber de exemplis Sacra Scripture de Nicolas Hanapis, copié en 1457 par Gaspar Poulet. Cet achat fut réalisé le 2 mars 1468 et Roland s'en défait du manuscrit cette même année en le vendant à l'évêque de Tournai, Guillaume Fillastre. Ce volume est aujourd'hui conservé à la bibliothèque de Saint-Omer sous la cote 169 . CATALOGUE générale des manuscrits des bibliothèques publiques des départements. Vol. III. Manuscrits de la bibliothèque Saint-Omer. Paris, Imprimerie Nationale: 1861, p. 91, ํo. 169; COMMENTAIRES de la Faculté de Médecine, p. 114, note 1; LOUIS, Nicolas - L'exemplus en pratiques. Production, diffusion et usages des recueils d'exempla latins aux $13^{e}-15^{e}$ siècles. Namur: Université de Namur, 2013, p. 232. Thèse de Doctorat.

50. L'emprunt se destinait à la correction de l'exemplaire que Roland avait de cette œuvre. FRANKLIN, Alfred - Les anciennes bibliothèques de Paris. Vol. I. Amsterdam: G. Th. Van Heusden, 1868, p. 52; DELISLE, Léopold - Le cabinet des manuscrits de la Bibliothèque Nationale. Vol. I. Paris: Imprimerie Nationale, 1881, p. 429, CHARMASSON, Thérèse - "Roland l'Écrivain", pp. 24-25.

51. DENIFLE, Henrich et al. - Auctarium Chartularii Universitatis Parisiensis: Liber procuratorum nationis Anglicanae (Alemanniae). Paris: Apud H. Didier, 1937, cols. 59, 64.

52. MARICHAL, Robert - Le Livre des prieurs de Sorbonne (1431-1485). Paris: Aux amateurs des Livres, 1987, p. 77; VIEILLARD, Jeanne; JULLIEN DE POMMEROL, Marie-Henriette - Le registre de prêt de la bibliothèque du Collège de Sorbonne de la Sorbonne (1402-1536). Paris: CNRS-Editions, 2000, pp. 36, 327, 679; KEYSER, Raphael de - "Individuel en collectief", p. 373. Il s'agit sans doute du De judiciis astrorum d' Hali Abenragel dont une copie subsiste à Paris, Bibliothèque Nationale, ms. Fr. 1352 en traduction française exécutée par Guillaume Harnoys en 1430, à la demande du duc de Bedford (BOUDET, Jean-Philippe; CHARMASSON, Thérèse - "Une consultation astrologique", pp. 258-259). Le manuscrit prêté serait très probablement l'une des œuvres suivantes répertoriées dans catalogue de la bibliothèque daté de 1338 (DELISLE, Léopold - Le cabinet des manuscrits, Vol. III, pp. $72,88,89)$.

53. VIEILLARD, Jeanne; JULLIEN DE POMMEROL, Marie-Henriette - Le registe, pp. 36, 328, 679. D'après Thérèse Charmasson, le manuscrit du Antidotarium était joint à une autre œuvre de SaintAmand intitulée Areole de conferentibus et nocentibus, maintenant conservée à la Bibliothèque Nationale de Paris (BNP, ms. latin 16 192). Voir DELISLE, Léopold - Le cabinet des manuscrits, Vol. III, pp. 66, 91; CHARMASSON, Thérèse - "Roland l'Écrivain", p. 25.

54. VIEILLARD, Jeanne; JULLIEN DE POMMEROL, Marie-Henriette - Le registe, pp. 36, 328, 679. Ce dernier faisait partie du noyau des livres enchainés de la magna libraria sous le titre Secretum magistri Hispani de morbis oculorum et curis eorum (CHARMASSON, Thérèse - "Roland l'Écrivain", p. 25).

55. Cette information est transmise par César Égasse du Boulay, à partir des registres de la nation de France (DU BOULAY, César Égasse - Remarques sur les bedeux de l'Université. Paris: Pierre de Bresche et Jacques Laize-de Bresche, 1670, p. 40). Il devrait s'agir du livre des procureurs de la nation contenant la relation séquentielle de ses réunions et le registre des événements attachés à son fonctionnement dont Du Boulay put encore consulter à l'aise au XVII ${ }^{\mathrm{e}}$ siècle et il ne reste aujourd'hui qu'un fragment concernant les années 1443 à 1456 . Pour cette question, voir VULLIEZ , Charles; VERGER, Jacques - "Cartulaires universitaires français”. in GUYOTJEANNIN, Olivier et al. (Eds.) - Les Cartulaires. Actes de la Table Ronde organisée par l'École Nationale des Chartes et le CNRS. Paris: École Nationale des Chartes, 1993, pp. 423-449; COURTENAY, William J. - "The Registers of the University of Paris and the Statutes against the Scientia Occamica". Vivarium 29/1 (1991), pp. 13-49, entre autres. 
56. Certains auteurs comme Danielle Jacquart contestent le fait, avancé par le même César Égasse $\mathrm{du}$ Boulay, selon lequel Roland avait été le recteur de l'Université de Paris en 1406-1407, avec l'argument qu'il il est entré à la faculté de Médicine seulement em 1419-1420, comme scholaris (DU BOULAY, César Égasse - Historia Universitatis Parisiensis. Vol. V. Paris: Pierre de Bresche et Jacques Laize-de Bresche, 1670, p. 919; JACQUART, Danielle - Le milieu médical, p. 264). Nous croyons que le sujet mérite davantage de la réflexion, une fois que la mention de 1419-1420 indique seulement qu'il avait passé à la faculté de Médicine, après ces études à la faculté d'Arts, où étaient recrutés d'ailleurs les recteurs de l'Université élus chaque quatre mois.

57. En 1414-1425, 1427-1428, 1428-1429 et 1429-1430.

58. Notamment le procès entre la faculté et le charlatan Jean Domprémi (COMMENTAIRES de la Faculté de Médecine, pp. 133, 137-140). Pour l'étude approfondie de celui-ci, voir GARRIGUE, Laurent - "Les professions médicales à la fin du Moyen Âge. L'exemple des praticiens parisiens devant le Parlement de Paris au XV $\mathrm{XV}^{\mathrm{e}}$ siècle". Bibliothèque de l'École des Chartes 156/2 (1999), pp. 317-367.

59. COMMENTAIRES de la Faculté de Médecine, pp. 136-137.

60. COMMENTAIRES de la Faculté de Médecine, p. 135 et ROUSE, Richard H.; ROUSE, Mary A. Illiterari et uxorati. Manuscripts and Their Makers: commercial Book Producers in Medieval Paris, 1200-1500. Vol. 2. Turnhout: Brepols, 2000, p. 134. Sur cette taverne, voir CHATELAIN, Émile "Notes sur quelques tavernes fréquentées par l'Université de Paris aux XIV et XV ${ }^{\mathrm{e}}$ siècles". Bulletin de la Société de l'histoire de Paris et de Île-de-France 25 (1898), p. 97.

61. COMMENTAIRES de la Faculté de Médecine, pp. 141-148.

62. Rappelons que son nom a été rayé de la liste des régents de l'année 1442-1443.

63. Par exemple, l'absentéisme des recteurs de la faculté de Médicine entre 1440 et 1442. COMMENTAIRES de la Faculté de Médecine, pp.167-169.

64. Il maintien quand-même une liaison avec sa faculté, puisqu'en 1449-1450, déjà installé à Bruges, il reçoit avec Jean Cotterau une missive envoyée par son ancienne faculté, possiblement sur la pratique illicite de la médicine dans cette ville. COMMENTAIRES de la Faculté de Médecine, p. 189.

65. Pour sa date critique, voir CHARMASSON, Thérèse - "L'Arithmétique de Roland", p. 176; BEAUJOUAN, Guy - "The Place of Nicolas Chuquet in a Typology of Fifteenth-Century Arithmetics”. in HAY, Cynthia (Ed.) - Mathematics from Manuscripts to print 1300-1600. Oxford: Clarendon Press, 1988, pp. 73-88; SZPIRGLAS, Jacques - Prosopographie des musiciens des SaintesChapelles de Paris (1248-ca1640) et de Bourges (1405-ca1640). Tours: Université François-Rabelais, 2015, p. 722.

66. Sur le caractère bibliophile du Duc, voir STRATFORD, Jenny - "The Manuscripts of John, Duke of Bedford: Library and Chapel". in WILLIAM, Daniel (Ed.) - England in the Fifteenth Century. Wooldbridge: Boydell Press, 1987, pp. 329-350.

67. DELISLE, Léopold - Le cabinet des manuscrits, Vol. II, p. 314. Le manuscrit Paris, Bibliothèque Nationale, fr. 24246 a été publié dans JACQUART, Danielle - "Hippocrates en français, le Livre des Amphorismes de Martim de Saint-Gille (1262-1363)". in JACQUART, Danielle - Les voies de la science grecque. Genève: Librairie Droz, 1997, pp. 241-329.

68. Pour la description et étude de cette source, voir POULLE, Emmanuel - "Horoscopes princiers des XIV et $\mathrm{XV}^{\mathrm{e}}$ siècles". Bulletin de la société des antiquaires de France (1969), pp. 63-69; BOUDET, Jean-Philippe et CHARMASSON, Thérèse - "Une consultation astrologique...", pp. 255-278; BOUDET, Jean-Philippe - Lire dans le Ciel. La bibliothèque de Simon de Phares, astrologue du XVe siècle. Bruxelles: Centre d'Études des Manuscrits, 1994, pp. 113-151.

69. En décembre 1468, en tant que conseilleur et physicien du duc, il reçoit la somme de 91 libres et seize soldes à cause de sa permanence à Bruxelles sous les ordres de son Seigneur et de la Duchesse et "pour certaines causes dont il ne veult plus ample declaracion icy estre faite". GREVE , Anke et al. - Comptes de l'argentier de Charles le Téméraire. Vol. I. Paris: De Boccard, 2001, p. 484, o․ 
2022. Une lettre d'Isabelle, datée de 1442, mentionne qu'elle avait envoyée Roland auprès du Seigneur de Croy, afin de guérir son petit-fils (SOMMÉ, Monique - La correspondance d'Isabelle de Portugal, duchesse de Bourgogne (1430-1471). Ostfildern: Jan Thorbecke Verlag, 2009, p. 153.

70. WICKERSHEIMER, Ernest - Dictionnaire biographique, p. 573. Sur les médecins du Duc, voir BAVEYE KOUIDRAT, Laurie - Exercer la médecine en milieu princier au XV'̀̀me siècle. L'exemple de la Cour de Bourgogne (1363-1482). Lille: Université Lille 3 - Charles de Gaulle, 2015.

71. Ces registres constituent les écrous des gages quotidiens versés aux membres de l'hotel ducal. CHARMASSON, Thérèse - "Roland l'Écrivain", pp. 24, 31. Sur cette source, voir KRUSE, Holger Hof, Amt und Gage. Die täglichen Gagenlisten des burgundischen hofes (1430-1467) und fer erste Hofstaat Karls des Kühnen (1456). Bonn: Bouvier Verlag, 1996.

72. BAVEYE KOUIDRAT, Laurie - Exercer la médecine, Vol. 2, p. 175-176. Nous remercions Sébastien Hamel de nous avoir remis cette liste il y a quelques années. Par manque d'espace, il n'est guère possible ici d'en faire l'exploitation et la publication. Cependant, elle demeure accessible sur www.prosopographia-burgundica.org.

73. CHARMASSON, Thérèse - "Roland l'Écrivain", p. 24. Les comptes de la maison ducale confirment ce montant annuel, comme celle de 1438-1439 (LABORDE, Le Comte de - Les ducs, p. 493, nº. 1901).

74. CHARMASSON, Thérèse - "Roland l'Écrivain", p. 24; LABORDE, Le Comte de - Les ducs, Vol. II, p. XI, note 2; BAVEYE KOUIDRAT, Laurie - Exercer la médecine, Vol. III, p. 331.

75. Il ne subsista aucune liaison épistolaire entre Roland et le Téméraire selon toute vraisemblance. Der Briefwechsel Karls des Kuhnen (1433-1477). Ed. Werner Paravicini et al. Frankfurt: Lang, 1995. 2 vols.

76. TROALEN, Phillipe - Charles le Téméraire, ses maladies et ses médecins. Paris: Université Paris Val-de-Marne, 1981, p. 112. Thèse de doctorat; BAVEYE KOUIDRAT, Laurie - Exercer la médecine, Vol. I, pp. 71, 150.

77. Pierre Michiel, valet de chambre et épicier du duc, paya six livres à maître Roland Lescripvain "pour certaines pommes pour odorer et sentir contre la pestillence". GREVE, Anke et al. - Comptes de l'argentier, p. 287; BAVEYE KOUIDRAT, Laurie - Exercer la médecine, Vol I, p. 218; Vol. III, pp. 331-334.

78. MARCHE, Olivier de la - Mémoires d'Olivier de la Marche., maître-d'hotel et capitaine des gardes de Charles de Téméraire. Ed. Henri Beaune et Jules D'Arbaumont. Vol. IV. Paris: Librairie Renouard, 1888, pp. 16-17.

79. DUBRULLE, Henry - "Les bénéficiaires des diocèses d'Arras, Cambrai, Thérouanne, Tournai sous le pontificat d'Eugène IV d'après les documents conservés aux Archives d'État, à Rome (suite) ". Analectes pour servir à l'histoire ecclésiastique de la Belgique, troisième série, t. 3, 33 (1907), p. 313, no 271; KEYSER, Raphael de - "Scrivers", col. 853.

80. CHARMASSON, Thérèse - "L'Arithmétique de Roland", p. 176.

81. La mention de cette supplique est faite seulement dans la supplique du 11 avril 1428. Il est possible que celle contenue dans de rotulus du Parlement de Paris n'ait pas été lancé dans les registres de la chancellerie apostolique, puisqu'à cette époque le registre de ces documents n'était plus systématique (WATT, Donald E.R. - "University Clerks and Rolls of Petitions for Benefices". Speculum 34 (1959), pp. 223-224).

82. COSTA, António Domingues de Sousa - Monumenta, pp. 263-264, nº. 1189.

83. DENIFLE, Henrich; CHATELAIN, Emile - Chartularium Universitatis Parisiensis, p. 433, no. 2240. Cette date est indiquée dans KEYSER, Raphael de - "Scrivers", col. 853.

84. COMMENTAIRES de la Faculté de Médecine, p. 141.

85. KEYSER, Raphael de - “Scrivers", col. 856.

86. KEYSER, Raphael de - "Scrivers", col. 856.

87. COSTA, António Domingues de Sousa - Monumenta, pp. 294-295, nº. 1221. 
88. DUBRULLE, Henry - "Les bénéficiaires", p. 313, nº 272; KEYSER, Raphael de - "Scrivers", col. 856; KEYSER, Raphael de - "Het boekenbezit", p. 23.

89. DUBRULLE, Henry - "Les bénéficiaires", p. 71, nº 264; FAYEN, A. - "Note sur un registre des Annates de la province de Reims sur Eugène IV (années 1431 à 1441)". Analectes pour servir à l'histoire ecclésiastique de la Belgique 35 (1909), p. 276, no. 91; KEYSER, Raphael de - "Scrivers", col. 854.

90. KEYSER, Raphael de - "Scrivers", col. 855. C'est ainsi que le duc de Bourgogne peut l'appeler de "prestre" dans la lettre de légitimation qu'il lui délivre en 1460. Sur ses fils, voir BAVEYE KOUIDRAT, Laurie - Exercer la médecine, Vol. III, p. 330-331.

91. Il ne réussit pas à avoir un bénéfice appartenant à la collation de l'Université et nous ne savons pas le résultat de sa pétition contenue dans le rotulus du parlement de Paris.

92. L'intercession ducale se prouve dans les deux cas.

93. Sur celle-ci, voir MOREAU, Édouard de - "Les familiers des ducs de Bourgogne dans les canonicats dans les anciens Pays-Bas”. in Mélanges d'histoire offerts à León Van der Essen (Miscellanea historica in honorem Leonis Van der Essen. Universitatis Catholicae in oppido Lovaniensi iam Annos XXV professoris. Bruxelles: Éditions universitaires, 1947, p. 435; MOLLAT, Michel - "Quatre missives d'Isabelle de Portugal, duchesse de Bourgogne". Revista Portuguesa da História 11-12 (1964), p. 6.

94. Ses longues absences à Arras, colmatées par la présence d'un vicaire, motivèrent toute de même un conflit avec l'Ordinaire à 1446 (GUESNON, A. - "Le Cartulaire de l'évêché d'Arras, manuscrit du XIII ${ }^{\mathrm{e}}$ siècle avec addition jusqu'au milieu du XVI ${ }^{\mathrm{e}}$, analysé chronologiquement”. Mémoires de l'Académie des Sciences, Lettres et Arts d'Arras. $2^{\mathrm{e}}$ série, 33 (1902), pp. 312, 314; DE LA FONS-MELICOCQ, Alexandre - "Les calligraphies et les manuscrits des cathédrales d'Arras, de Tournai, et de l'abbaye de Saint-Bertin". Revue du Nord de la France 1 (1854), p. 22.

95. Ce même argument a été utilisé par le chapitre de saint-Donatien pour lui empêcher d'accéder du décanat lors de cette même année de 1439, une action qui s'est avéré futile étant donné l'intercession qu'il bénéficia de la famille ducale (PEARSON, Andrea - Gender in Burgundian Devotional Art, 1350-1530. Experience, Authority, Resistance. Aldershot: Ashgate, 2005, p. 134). Il est possible parfois d'entrevoir ses actions en tant que doyen de cette institution: SCHREVEL, A. C. de. - Histoire du séminaire de Bruges. Vol. I. Bruges: Imprimerie A. de Zuttere-van Kersschaver, 1895, p. 117 (doc. de 1455); BERLIÈRE, Ursmer - Monasticon Belge. Vol. III. Bruges: Centre Nationale de Recherches d'histoire religieuse, 1978, p. 1137 (doc. de 1461); GAILLIARD, Edward et GILLODTSVAN SEVEREN, Louis - Inventaire des archives de la ville de Bruges. Bruges: E. Gailliard, 1876, p. 545 (doc de 1468).

96. À cette fin, il participa à deux reprises, en 1455 et 1460, dans des commissions médicales servant à évaluer la condition pestifère de deux malades à la léproserie de saint-Pierre de Bruxelles.

97. À ce qu'il paraît, à l'us qu'il faisait de l'hydromel. DOORSLAER, G. Van - "Traitement de la lithiase rénale en 1490". Yperman: Bulletin de la Société belge de l'histoire de la médecine. Bulletin der belgische vereeniging voor geschiedenis der geneeskunde 1 (1924), pp. 121-122 et WICKERSHEIMER, Ernest - Dictionnaire biographique, p. 724.

98. Cet événement est décrit dans le livre des procureurs de la nation. DENIFLE, Henrich et al. Auctarium Chartularii, col. 372. 


\section{RÉSUMÉS}

Cet article vise l'étude biographique d'un médecin portugais du $\mathrm{XV}^{\mathrm{e}}$ siècle, personnage encore insuffisamment connu de l'historiographie portugaise. Partant des faits connus et d'autres inédits, on propose une mise en perspective de son parcours entre l'Université, le service des Grands et le milieu ecclésiastique, constituant ainsi un cas de mobilité institutionnelle et géographique d'un médecin à la fin du Moyen Age.

This article aims at the biographical study of a Portuguese physician of the fifteenth century, still insufficiently known by the Portuguese historiography. Based on known and unpublished facts, it proposes a perspective into his public life, shared between the University, the service of the Great and the ecclesiastical environment, thus constituting a case of institutional and geographical mobility of a physician at the end of the Middle Ages.

\section{INDEX}

Mots-clés : Université de Paris, Médicine médiévale, Cour de Bourgogne, prêts de libres, Bruges Keywords : University of Paris, medieval medicine, Burgundy Court, books loans, Bruges

\section{AUTEUR}

\section{MÁRIO FARELO}

Instituto de Estudos Medievais, Faculdade de Ciências Sociais e Humanas, Universidade Nova de Lisboa, 1061-061, Lisboa, Portugal

Centro de Estudos de História Religiosa, Faculdade de Teologia, Universidade Católica

Portuguesa, 1649-023, Lisbon, Portugal

Centro de História da Universidade de Lisboa, Faculdade de Letras - Universidade de Lisboa,

1600-214, Lisboa, Portugal

mario.farelo@fcsh.unl.pt

https://orcid.org/0000-0002-2905-6564 